\title{
Demonstration of a Reflectarray with Near-field Amplitude and Phase Constraints as Compact Antenna Test Range Probe for 5G New Radio Devices
}

\author{
Álvaro F. Vaquero, Manuel Arrebola, Senior Member, IEEE, Marcos R. Pino, Rafael Florencio, and José
} A. Encinar, Fellow, IEEE.

\begin{abstract}
In this work a reflectarray is proposed to be used as a probe of a reduce and portable Compact-Antenna-TestRange for 5G new radio devices. The reflectarray works at $28 \mathrm{GHz}$ and produces a quiet zone in the near-field region of the antenna. Considering that the quiet zone specifications are established in terms of the amplitude and phase ripple, a synthesis technique is presented to optimize the near-field in the Fresnel region of a reflectarray with amplitude and phase constraints. The proposed technique is based on the generalized Intersection Approach, using the Levenberg-Marquardt algorithm as its backward projector obtaining a novel technique in near-field synthesis. This technique is applied to improve the quiet zone radiated by the proposed reflectarray, overcoming the amplitude and phase limitations of the initial configuration. The solution provided by this process is used to design and manufacture a reflectarray based on a three-parallel-dipole cell. Finally, the prototype is measured in a near-field planar range facility in order to evaluate the radiated quiet zone and demonstrate the methodology. The prototype satisfies the tight requirements in amplitude and phase, obtaining promising results.
\end{abstract}

Index Terms-Near-field synthesis, compact antenna test range, CATR, reflectarray, measurement system.

\section{INTRODUCTION}

$\mathbf{T}$ HROUGHOUT the last decades antenna measurement systems have been constantly developed in order to adapt them to the new technological requirements. In a first approach, far-field ranges were used to directly measure the radiation pattern of the Antenna Under Test (AUT). Since the AUT should be placed in the far-field of the probe, these

This work was supported in part by the Ministerio de Ciencia, Innovación y Universidades under project TEC2017-86619-R (ARTEINE), by Ministerio de Economía, Industria and Competitividad under project TEC2016-75103C2-1-R (MYRADA), and by the Gobierno del Principado de Asturias/FEDER under Project GRUPIN-IDI/2018/000191.

A. F. Vaquero, M. Arrebola, and M. R. Pino are with the Department of Electrical Engineering, Group of Signal Theory and Communications Universidad de Oviedo, Gijón 33203, Spain (e-mail: fernandezvalvaro@uniovi.es; arrebola@uniovi.es; mpino@uniovi.es).

R. Florencio is with the Department of Physics and Mathematics, University of Alcalá, 28805 Alcalá de Henares, Spain (e-mail: rafael.florencio@uah.es). J.A. Encinar is with the Information Processing and Telecommunications Center, Universidad Politécnica de Madrid, Madrid, 28040, Spain (e-mail: jose.encinar@upm.es).

Color versions of one or more of the figures in this paper are available online at http://ieeexplore.ieee.org.

Digital Object Identifier XX.XXXX/TAP.XXXX.XXXXXXX ranges usually require large dimensions, which leads to really bulky systems. Conversely, the near-field scanning ranges only measure the near-field of the AUT [1], therefore the system dimensions are notably reduced. However, a large amount of near-field data has to be acquired in order to perform a Near-Field to Far-Field (NF-FF) transformation to get the field value, demanding long time measurements and a high stable measurement system.

Compact Antenna Test Ranges (CATR) [2] are an attractive alternative to replace far- and near-field scanning ranges. This solution provides a system with the ability of measuring the radiation pattern directly, even though the AUT is within the near-field region of the probe. These systems are based on the existence of an area within the Fresnel region of the probe known as quiet zone. Such area virtually behaves as an uniform plane wave, and is suitable for antenna measurements in a relatively compact space.

On the other hand, new technological needs have appeared together with the popularity of the (sub)millimeter band applications. Particularly, the deployment of $5 \mathrm{G}$ communications in the three bands of 28, 39 and $60 \mathrm{GHz}$ [3], [4], [5], automotive radars at $77 \mathrm{GHz}$ [6], [7] or space communications [8], [9] among others. Hence, antenna measurement systems need to evolve and fulfill these new requirements, where CATRs are a suitable candidate to test these new devices. These systems are traditionally based on a parabolic reflector configuration, which provides a solution that collimates the incoming field radiated by its feed, that typically is a horn, in a certain direction where the quiet zone can be found. The geometrical properties of the parabolic reflector allows to directly obtain a plane wave, whilst the amplitude is conditioned by the amplitude taper of the incident field. However, additional drawbacks appears at those frequencies. Mainly, parabolic reflectors present particular issues in terms of their size or manufacturing process, which needs low surface errors and therefore increase the expenses.

In [10] and [11] the use of dielectric lenses was studied as an alternative to overcome the reflector drawbacks, though they do not obtain a sufficient surface accuracy. Hereinafter, it was introduced the concept of a hologram-based CATR [12]. Mainly, a planar hologram is used to provide a solution similar 
to parabolic reflectors, where this planar structure behaves as the collimating element of an incoming field provided by a feed. Then, the quiet zone may be found in the radiated plane wave. The advantage of using holograms regards on their lowcost and easy manufacturing process, reducing the surface accuracy requirements. This proposal has clearly demonstrated good performances in (sub)millimeter bands, even when it is compared with a planar near-field scanning [13].

Regarding the quiet zone performances, it should be highlighted that its size directly depends on the equivalent aperture $(D)$ of the probe, leading to use large electrical antennas and, thus bulky probes. i.e. in [13] a hologram of more than one meter in each direction is used to get a quiet zone of $70 \mathrm{~cm} \times 45 \mathrm{~cm}$ equivalent to a $46 \% \times 32 \%$ of the hologram aperture. Beside that, a near-field volume is theoretically considered quiet zone whenever its maximum ripple in amplitude and phase is lower than $1 \mathrm{~dB}$ and $10^{\circ}$ respectively. However, it can be found implemented systems in that are operative with higher peak-to-peak ripples [13]. Slight increments on the amplitude ripple have small effects on the radiation pattern measurement, while errors in the phase distort the gain and sidelobe levels or fill the nulls [14].

Another potential alternative to reflectors and holograms is the reflectarray antenna, which also has the capacity to transform a spherical wave into a plane wave, wherein the quiet zone may be located. Reflectarray antennas are planar structures based on printed technology, which reduces the surfaces error requirements and the manufacturing costs. These antennas have widely demonstrated good performances in multiple applications with tight specifications, such as satellite communications, specially Direct Broadband Satellite (DBS) missions [15], innovative wireless communications [16] or femtocells for $5 \mathrm{G}$ indoor communications [17]. In [18] the quiet zone radiated by a reflectarray is evaluated and improved using a near-field synthesis in order to reduce both amplitude and phase ripples. This synthesis is based on a direct optimization technique that, despite reaching acceptable performance on the quiet zone, the obtained phase variation along the reflectarray surface is too high for a potential implementation. This leads to a main drawback of these techniques, the degree of freedom of their solutions and their hardly implementation into a design. In addition, there is a strong limitation in the number of optimization variables that can be managed. One approach to overcome these issues is the generalized Intersection Approach for far-field synthesis, which can efficiently deal with a large amount of optimization variables [19], [20], which also can be related with the antenna geometry. Thus, the layout of the antenna can be considered into the optimization process based on Intersection Approach, leading to more feasible solutions than the reached in classic direct optimization techniques. In [21] the Intersection Approach is introduced to synthesize the near-field radiated by a reflectarray but just with amplitude constraints, resulting in a degradation of the phase front.

In this work, the generalized Intersection Approach is extended to synthesised the near-field with both amplitude and phase constraints, by only optimizing the phase distribution of the reflection coefficients of the reflectarray elements.
Then, a squared reflectarray comprises by $44 \times 44$ elements $\left(188.76 \times 188.76 \mathrm{~mm}^{2}\right)$ is proposed to generate a quiet zone at $500 \mathrm{~mm}(46.6 \lambda)$ with a working frequency of $28 \mathrm{GHz}$. The width of the quite zone is $100 \mathrm{~mm}$, the height is $100 \mathrm{~mm}$, equivalent to more than a $53 \%$ of the antenna aperture width in the $x$ and $y$ axes, which is obtained through a phase-only synthesis including near-field amplitude and phase constraints, using the generalized Intersection Approach. The obtained phase distribution reaches to satisfy the quiet zone requirements and it is suitable to perform a design. The elementary reflectarray element is based on three parallel dipoles, which obtain an enough phase-shift to cover a $360^{\circ}$ range [22]. The design is performed and measured in a planar range facility to evaluate the generated quiet zone. Finally, measurements are analysed to validate the proposed technique and the utility of reflectarrays as a probe in a small and portable CATR systems for $5 \mathrm{G}$ new radio devices measurements.

\section{INTERSECTION APPROACH FOR NEAR-FIELD SHAPING}

\section{A. Classical Intersecion Approach}

Unlike the classical optimization algorithms, where the algorithm looks for the minimization of a cost function, the Intersection Approach (IA) searches the intersection between two sets. Those sets are defined as: 1) the set of the fields that complies the desired specifications $(\mathcal{M})$, and 2) the set within all the field that can be generated by the antenna $(\mathcal{R})$. This intersection search is based on an iterative process of alternative projections, which are performed by two operations in each iteration:

$$
\vec{E}_{N F}^{i+1}=\mathcal{B}\left[\mathcal{F}\left(\vec{E}_{N F}^{i}\right)\right]
$$

where $\mathcal{F}$ is the forward projector, which is used to project a point of $\mathcal{R}$ onto $\mathcal{M} ; \mathcal{B}$ is the backward projector that allows to retrieve from one point of $\mathcal{M}$ onto $\mathcal{R}$; and $\vec{E}_{N F}$ is the field radiated by the antenna at the $i$-iteration. The ideal results of this iterative process are a field that belongs concurrently to both sets, therefore obtaining a field within the specifications but also feasible to be radiated by the antenna geometry.

The Intersection Approach concept can be also explained in terms of the tangential field at the aperture $\left(\vec{E}_{A P}\right)$ and its radiated fields $\left(\vec{E}_{N F}\right)$. If a single-offset reflectarray configuration is taken as reference (see Fig. 1), and the flowchart shown in Fig. 2 is followed, the electric tangential field at the reflectarray aperture is computed as:

$$
\vec{E}_{A P}\left(\vec{r}_{m}\right)=\mathbf{R}^{m} \cdot \vec{E}_{i n c}\left(\vec{r}_{m}\right)=\left(\begin{array}{cc}
\rho_{x x}^{m} & \rho_{x y}^{m} \\
\rho_{y x}^{m} & \rho_{y y}^{m}
\end{array}\right) \cdot\left(\begin{array}{c}
E_{x, i n c} \\
E_{y, i n c}
\end{array}\right)
$$

where $\mathbf{R}$ is the reflection coefficient matrix that relates the complex amplitude of the tangential components of the incident field, $E_{x, \text { inc }}$ and $E_{y, i n c}$, and the reflected electric field by the $m$ th-element of the reflectarray located at the point $\vec{r}_{m}$. This reflection matrix is obtained in each reflectarray cell under local periodicity assumption [23], and its elements are the direct reflection coefficients $\left(\rho_{x x}^{m}\right.$ and $\left.\rho_{y y}^{m}\right)$ and the cross reflection coefficients $\left(\rho_{x y}^{m}\right.$ and $\left.\rho_{y x}^{m}\right)$. 


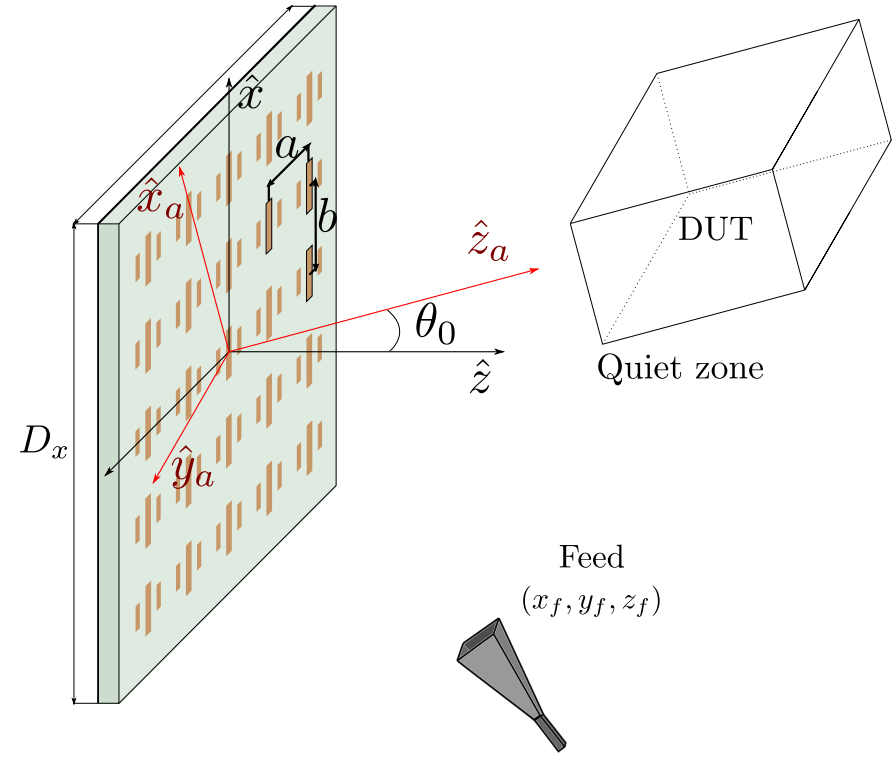

Fig. 1: Scheme of a CATR system based on an offset configuration reflectarray antenna.

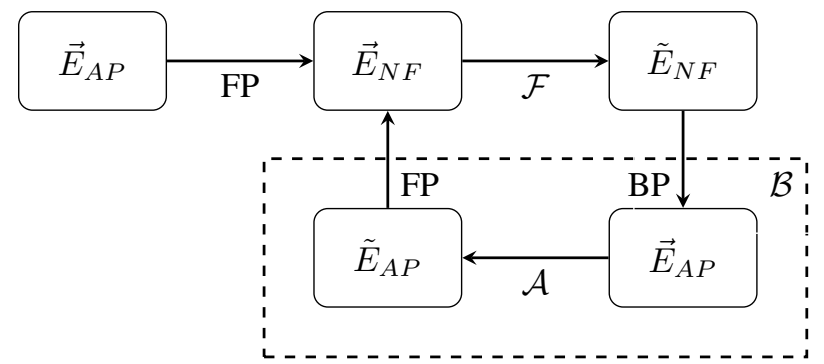

Fig. 2: Flowchart of the classical Intersection Approach.

However, in (2) when dealing with a Phase-Only Synthesis (POS) of the reflectarray element, some simplifications may be considered. Due to the reflectarray unit cells are modelled as an ideal phase shifter, the module of the reflection coefficients is assumed to be 1, therefore no losses are considered (i.e. $\left.\left|\rho_{x x}^{m}\right|=\left|\rho_{y y}^{m}\right|=1\right)$. Additionally, there is no cross-polarization influence $\left(\rho_{x y}^{m}=\rho_{y x}^{m}=0\right)$. Now, the coefficient reflection matrix is simplified to

$$
\mathbf{R}^{m}=\left(\begin{array}{cc}
e^{j \phi_{x x}^{m}} & 0 \\
0 & e^{j \phi_{y y}^{m}}
\end{array}\right)
$$

where $\phi_{x x}^{m}$ and $\phi_{y y}^{m}$ are the phase of the direct reflection coefficients of the element, which are used as degrees of freedom in the synthesis of the near-field radiated by the reflectarray.

Once the field at the aperture is computed, the near-field $\vec{E}_{N F}$ can be obtained by forward propagation (FP). In this work, this propagation model is based on a principle of superposition, where each element of the reflectarray is an unit radiation element modelled as a small aperture antenna, instead of a punctual isotropic source. Then, the near-field radiated by the reflectarray at one point of the space is computed as the sum of all the far-field contributions radiated by each reflectarray cell:

$$
\vec{E}_{N F}(\vec{r})=\sum_{m=1}^{N \text { elem }} \vec{E}_{F F}^{m}(\vec{r})
$$

where $\vec{r}$ is the position vector where the near-field of the reflectarray is computed; Nelem is the total number of unit radiation elements; and $\vec{E}_{F F}^{m}(\vec{r})$ is the far-field radiated by the $m$-element at $\vec{r}$. This far-field contribution of the $m$ element can be computed using the Second Principle of Equivalent for planar aperture antennas, which uses the field at the aperture $\vec{E}_{A P}$ of (2) [24].

Typically, the computed near-field $\left(\vec{E}_{N F}\right)$ does not satisfies the specifications, therefore the forward projector $(\mathcal{F})$ projects it onto a new radiated field $\left(\tilde{E}_{N F}\right)$ that fulfills the requirements. However, this does not necessarily means having a radiated field that can be radiated by the antenna, since the constraints are only imposed in the radiated field but not in the antenna optics. The backward projector $(\mathcal{B})$ is more complex than the forward and is divided into several steps. Firstly, backward propagation (BP) allows to compute the source of $\tilde{E}_{N F}$, thus the field at the aperture $\vec{E}_{A P}$ is calculated. Reminding that the field at the aperture depends on the element response (see (2)), it is not ensured that $\vec{E}_{A P}$ can be obtained with the proposed antenna. Hence, new constraints $(\mathcal{A})$ have to be imposed on the antenna geometry in order to obtain a achievable field at the aperture $\left(\tilde{E}_{A P}\right)$. Although the forward propagation of this new field is not $\tilde{E}_{N F}$, the nearfield obtained is closer to the requirements than the starting point, and additionally it is guaranteed that is achievable by the antenna optics. Doing this process iteratively, it may be reached a solution that satisfies both geometry and radiated field conditions.

\section{B. Generalized Intersection Approach}

In the classic implementation, the hardest step to deal with is the backward projector, particularly the need to find a proper backward propagation definition. In order to avoid it, there is an alternative that proposes the use of an optimization algorithm instead [25]. In this present case, the LevenbergMarquardt Algorithm (LMA) has been chosen to be integrated inside the Intersection Approach as Fig. 3 shows. This algorithm is used to find $\vec{E}_{N F}^{i+1}$ in $\mathcal{R}$ closer to $\tilde{E}_{N F}$ than $\vec{E}_{N F}$.

Let us suppose a field at the aperture $\vec{E}_{A P}$ radiated by the antenna. Then, the previous forward propagation (FP) is applied and a generated field within the near-field region is obtained $\left(\vec{E}_{N F}\right.$ belonging to $\left.\mathcal{R}\right)$. It is likely that on the first iteration this field does not fulfill the requirements, therefore the forward projector $(\mathcal{F})$ will project it onto a desired field $\left(\tilde{E}_{N F}\right)$, which belongs to the set $\mathcal{M}$. This projector is defined to ensure that:

$$
T_{\text {lower }} \leq \tilde{E}_{N F} \leq T_{\text {upper }}
$$

where $\tilde{E}_{N F}$ is the constrained field that belongs to $\mathcal{M} ; T_{\text {lower }}$ and $T_{\text {upper }}$ are the lower and upper specification boundaries respectively. 


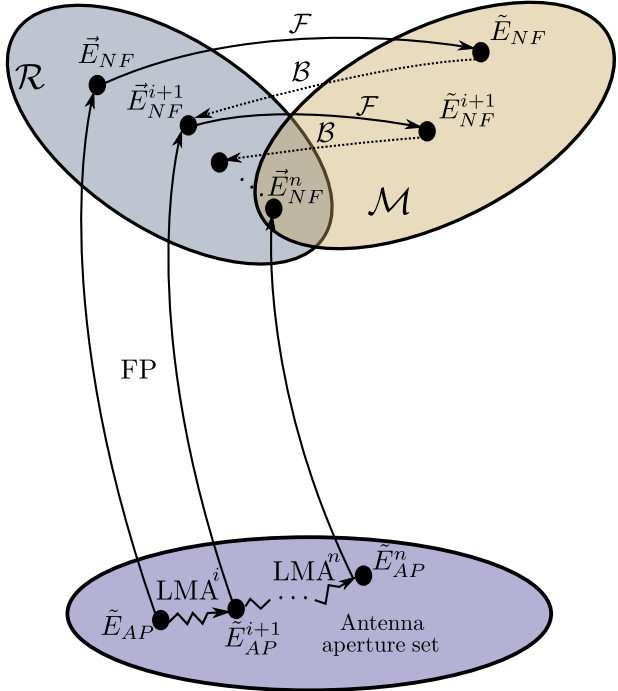

Fig. 3: Sketch of the generalized Intersection Approach and its iterative process.

Ultimately, this projector trims the radiated field according to certain specifications, which are generally given in form of templates. Hence, $\tilde{E}_{N F}$ can be computed as

$$
\tilde{E}_{N F}(\vec{r})= \begin{cases}T_{\text {upper }}(\vec{r}), & \text { if } \vec{E}_{N F}(\vec{r}) \geq T_{\text {upper }}(\vec{r}) \\ T_{\text {lower }}(\vec{r}), & \text { if } \vec{E}_{N F}(\vec{r}) \leq T_{\text {lower }}(\vec{r}) \\ \vec{E}_{N F}(\vec{r}), & \text { otherwise }\end{cases}
$$

To apply the optimization algorithm LMA, the generalized Intersection Approach defines a functional $d$ which evaluates the distances from one point of $\mathcal{R}$ to another of $\mathcal{M}$, thus the distance from $\vec{E}_{N F}$ to $\tilde{E}_{N F}$ (see Fig. 3). This functional is based on the Euclidean distance definition but, it should be kept in mind that each element of those sets has to be expressed in terms of magnitude and phase. Therefore, the functional should considered both components independently.

$$
\begin{aligned}
d=d_{M}+d_{P}= & \int_{\Omega} w_{M}\left(\left|\tilde{E}_{N F}\right|^{2}-\left|\vec{E}_{N F}^{\mathrm{LMA}}\right|^{2} \mid\right) d \Omega+ \\
& \int_{\Omega} w_{P}\left(\left|\angle \tilde{E}_{N F}-\angle \vec{E}_{N F}^{\mathrm{LMA}}\right|\right) d \Omega
\end{aligned}
$$

where $\Omega$ is the volume where the near-field is computed; and $w_{M}$ and $w_{P}$ are weight functions. If this volume is discretized in $N_{z}$ planes along $\hat{z}$ with $N_{x y}$ point each one, this functional can be expressed as

$$
\begin{aligned}
d & =\sum_{l}^{N_{z}}\left[\sum_{j}^{N_{x y}} w_{M}\left(\left|\tilde{E}_{N F, j}\right|^{2}-\left|\vec{E}_{N F, j}^{\mathrm{LMA}}\right|^{2} \mid\right)+\right. \\
& \left.+\sum_{j}^{N_{x y}} w_{P}\left(\left|\angle \tilde{E}_{N F, j}-\angle \vec{E}_{N F, j}^{\mathrm{LMA}}\right|\right)\right] \Delta_{x, j} \Delta_{y, j}
\end{aligned}
$$

where the subscript $l$ indicates the plane that is evaluated and $j$ the $(x, y)$ point where the distance is computed; and $\Delta_{x, j}$ and $\Delta_{y, j}$ are the step of the plane discretization. $\vec{E}_{N F}^{\mathrm{LMA}}$ is the field used by the LMA to evaluate $d$, which is related with the field at the aperture as $\operatorname{FP}\left(\vec{E}_{A P}^{\mathrm{LMA}}\right)$, being the degree of freedom the phase distribution of the reflection coefficients on the reflectarray surface $\left(\phi_{x x}\right.$ or $\left.\phi_{y y}\right)$.

Note that, the goal of the LMA is only to reduce $d$ in each iteration of the generalized Intersection Approach, but not reach a minimum of this distance, thus only few iterations are needed [25]. The starting point of the LMA is the near-field of the iteration $i$ of the generalized Intersection Approach $\left(\vec{E}_{N F}^{i}\right)$ and provides the near-field of the iteration $i+1\left(\vec{E}_{N F}^{i+1}\right)$, closer to $\tilde{E}_{N F}$. After several iterations of generalized Intersection Approach, an intersection between both sets may be found, $\mathcal{M}$ and $\mathcal{R}$, obtaining a near-field within the constraints and feasible by the antenna. If this intersection is not possible, at least to obtain a field $\vec{E}_{N F}$ with the least distance to $\tilde{E}_{N F}$.

Due to the LMA is a gradient-based algorithm, the differential contributions technique (DFC) [26] can be applied. This notably speeds up the computation of the functional gradient reaching computing times similar to analytical derivatives, whenever are available [27].

\section{REFLECTARRAY DEFINITION}

The proposed antenna to demonstrate its use as CATR probe is a squared reflectarray made up of $44 \times 44$ (1936) elements, which are distributed in a regular grid of a periodicity of $4.29 \times 4.29 \mathrm{~mm}^{2}$ along the $\mathrm{x}-$ and $\mathrm{y}$-axis. In this case, the reflectarray is feed using an offset configuration as shown in Fig. 1. The center phase of the feed is placed at $\left(x_{f}, y_{f}, z_{f}\right)=$ $(-79.3,0,200) \mathrm{mm}$ considering the centre of the reflectarray as the origin of the system of coordinates. This feed is a pyramidal standard horn gain of $15 \mathrm{dBi}$ gain (Narda v639), which generates an illumination taper of $-15.80 \mathrm{~dB}$ at the edge of the reflectarray at a working frequency of $28 \mathrm{GHz}$.

On the other hand, the proposed unit cell to design the reflectarray is based on a central dipole and two lateral ones that are separated at a distance $S=1.43 \mathrm{~mm}$ from the central dipole as Fig. 4 depicts. The dipoles work in linear polarization and are oriented according to the $\mathrm{x}$-axis of the reflectarray. The width of the three dipoles is $W=0.5 \mathrm{~mm}$ whilst the lateral dipoles lengths $\left(L_{1}\right)$ are a function of the central length $L_{2}$ as $L_{1}=0.7 L_{2}$. The dipoles are printed on the same size of a $0.762 \mathrm{~mm}$ thickness $(h)$ Diclad 880 substrate $\left(\epsilon_{r}=2.3, \tan \delta=0.005\right)$.

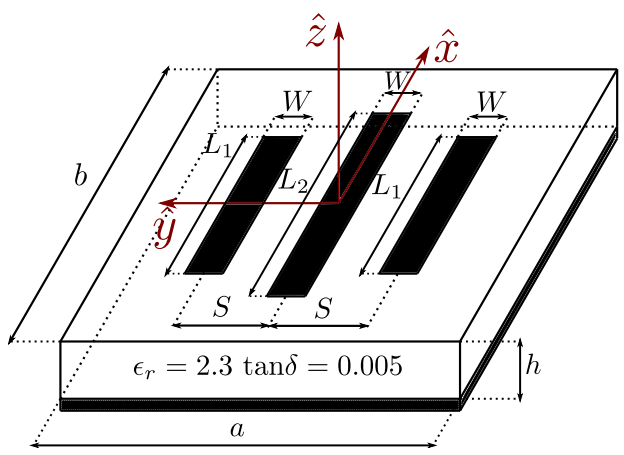

Fig. 4: Reflectarray element based on three parallel dipoles for a single polarization.

The phase responses of the cell are obtained by the variation of the length $L_{2}$. In Fig. 5 both amplitude and phase response 


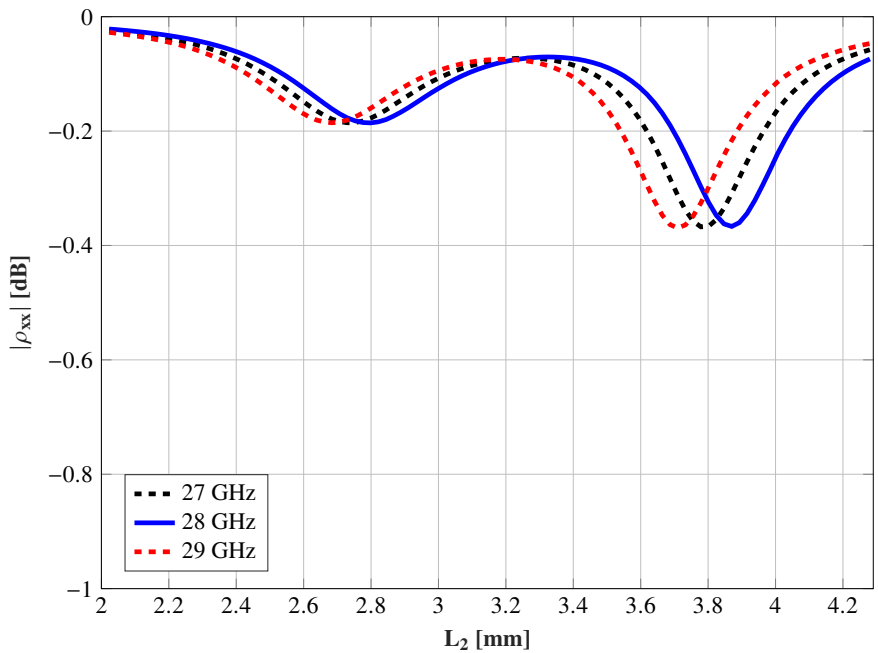

(a)

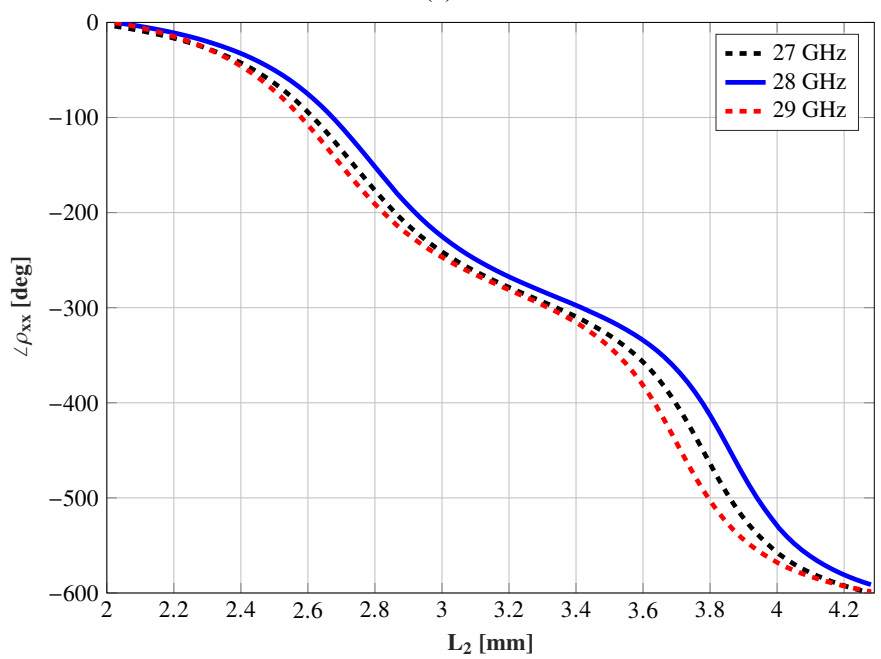

(b)

Fig. 5: Reflectarray cell characterization in x-pol versus $L_{2}$ (length of the central dipole) for incident (a) amplitude (b) phase.

are shown for normal incidence, obtaining a phase-shift larger than $360^{\circ}$. In addition, the studied range presents a soft slope, which helps to minimize the possible phase-shift errors due to the manufacturing tolerances. The amplitude in the worst case is lower than $-0.40 \mathrm{~dB}(>0.91$ of reflection) and this cell response is stable within a $2 \mathrm{GHz}$ bandwidth regarding the central frequency. The angular stability of the cell response has been validated through simulations.

Then, a reflectarray is defined to point at $\hat{z}_{a}$ direction with an angle $\theta_{0}=20^{\circ}$ that generates an equivalent aperture $(D)$ equivalent to

$$
\begin{aligned}
D= & D_{x} \times D_{y}=188.76 \mathrm{~mm} \cdot \cos \theta_{0} \times 188.76 \mathrm{~mm}= \\
& 177.36 \times 188.76 \mathrm{~mm}^{2}
\end{aligned}
$$

The quiet zone is evaluated on a perpendicular plane to $\hat{z}_{a}$ direction at $500 \mathrm{~mm}(46.66 \lambda)$ from the centre of the reflectarray, which provides a very compact structure since the far-field region starts at $12.52 \mathrm{~m}(1170.40 \lambda)$. The quiet zone specifications are defined in a circular area of diameter

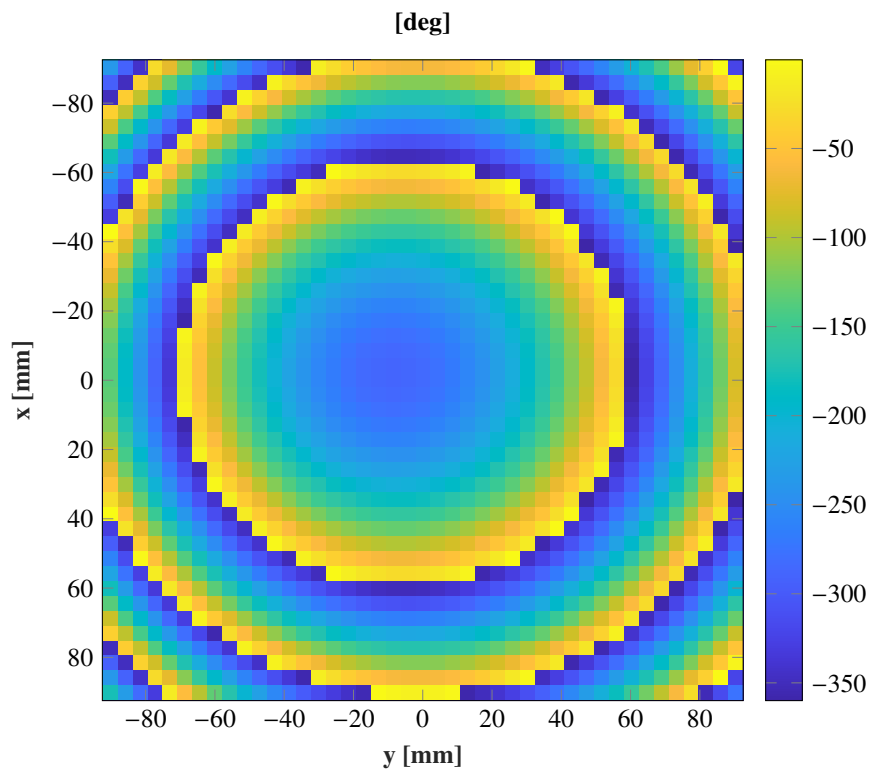

Fig. 6: Initial phase distribution [deg] of a far-field focused reflectarray at $28 \mathrm{GHz}$.

$100 \mathrm{~mm}(9.33 \lambda)$, more than the $56 \%$ and $53 \%$ of the antenna aperture width in the $x$ and $y$ axis, respectively. The maximum ripple within this area is $1 \mathrm{~dB}$ and $10^{\circ}$ in amplitude and phase respectively, thus the theoretical quiet zone definition.

\section{NEAR-FIELD SYNTHESIS AND DESIGN BOTH AMPLITUDE AND PHASE CONSTRAINTS}

\section{A. Starting point}

Considering the geometry previously defined, the starting phase distribution of the reflection coefficients $\left(\rho_{x x}\right)$ selected is that defined for a far-field focused reflectarray radiating a pencil beam in a pointing direction $\left(\theta_{0}, \phi_{0}\right)=\left(20^{\circ}, 0^{\circ}\right)$. This phase distribution can be analytically computed as

$$
\phi_{r}\left(x_{m}, y_{n}\right)=-k_{0} x_{i} \sin \theta_{0}-\phi_{i n c}\left(x_{m}, y_{n}\right)
$$

where $\phi_{i n c}$ is the incident field phase provided by the feed at the $(m, n)$ th element; and $k_{0}$ is the vacuum wavenumber. Fig. 6 shows the initial phase distribution computed using (10).

The quiet zone obtained by this phase distribution is shown in Fig. 7 for both amplitude and phase, additionally the quiet zone boundaries are represented using the solid red line. Due to the near-field is collimated in the direction where the quiet zone is placed, the phase of this field behaves as almost flat, specially at the $x=0$. The offset cut $(y=0)$ increases the ripple because of the non-symmetry, even so, the maximum peak-to-peak ripple in the whole area is $22^{\circ}$. Conversely, the amplitude has a worse behaviour considering the ripple specifications. According to (2), the reflected field depends on the field provided by the feed, therefore if the field at the reflectarray surface has a high amplitude taper, the quiet zone will be affected increasing the variation along the zone more than $8 \mathrm{~dB}$ as Fig. 7 a shows. 


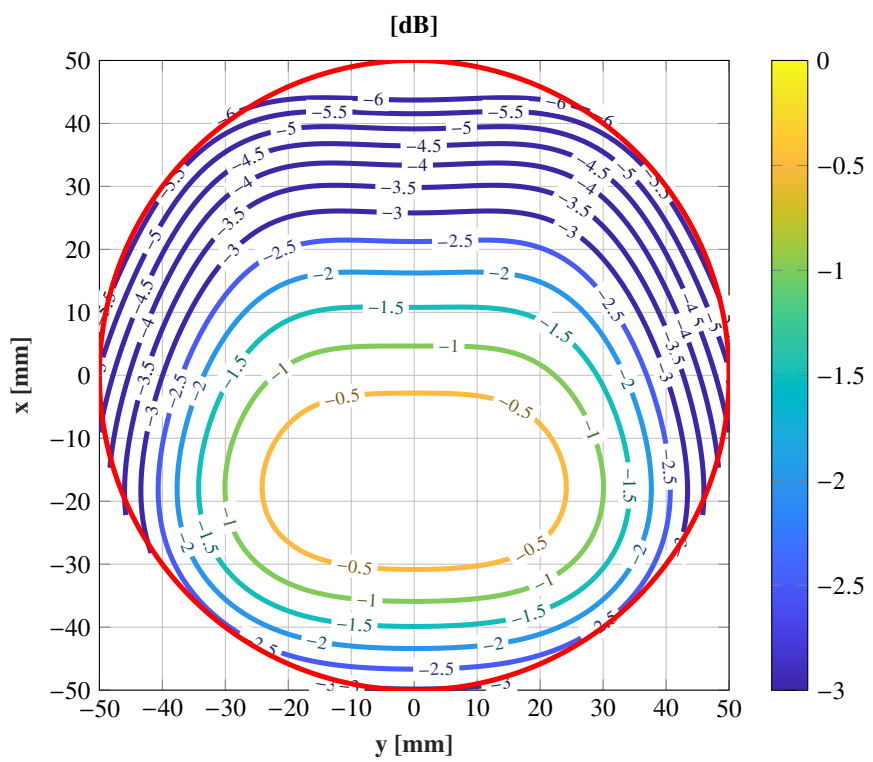

(a)

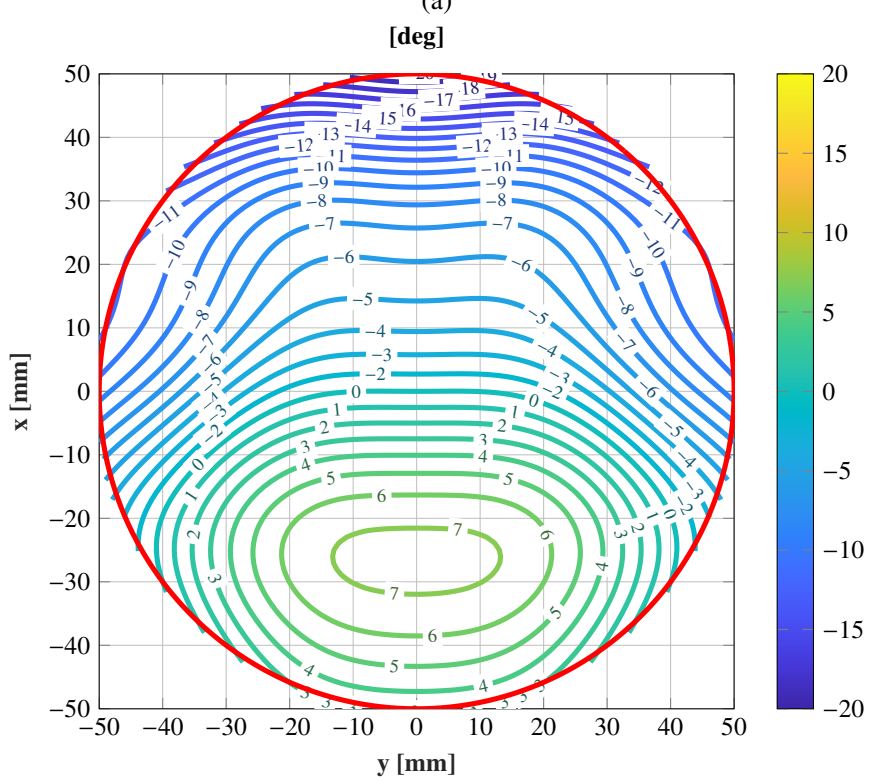

(b)

Fig. 7: Quiet zone for the starting point. (a) Normalized amplitude (dB) to the maximum. (b) Normalized phase (deg) to the value at the centre of the zone.

\section{B. Synthesis results}

The optimization process has been divided into a multistage strategy, particularly a total of 8 stages were needed until reaching the final solution. Starting from $1.5 \mathrm{~dB}$ and $20^{\circ}$ of amplitude and phase ripple, the specifications have been tightened once the previous stage requirement had been fulfilled. The $1 \mathrm{~dB}$ and $10^{\circ}$ ripple has been virtually reached after 2938 iterations of the generalized Intersection Approach and 3 LMA iterations in each one. The phase distribution of the reflectarray elements obtained is shown in Fig. 8. The phase variation along the reflectarray surface is smooth enough for a proper design.

The synthesised quiet zone is shown in Fig. 9, while Table

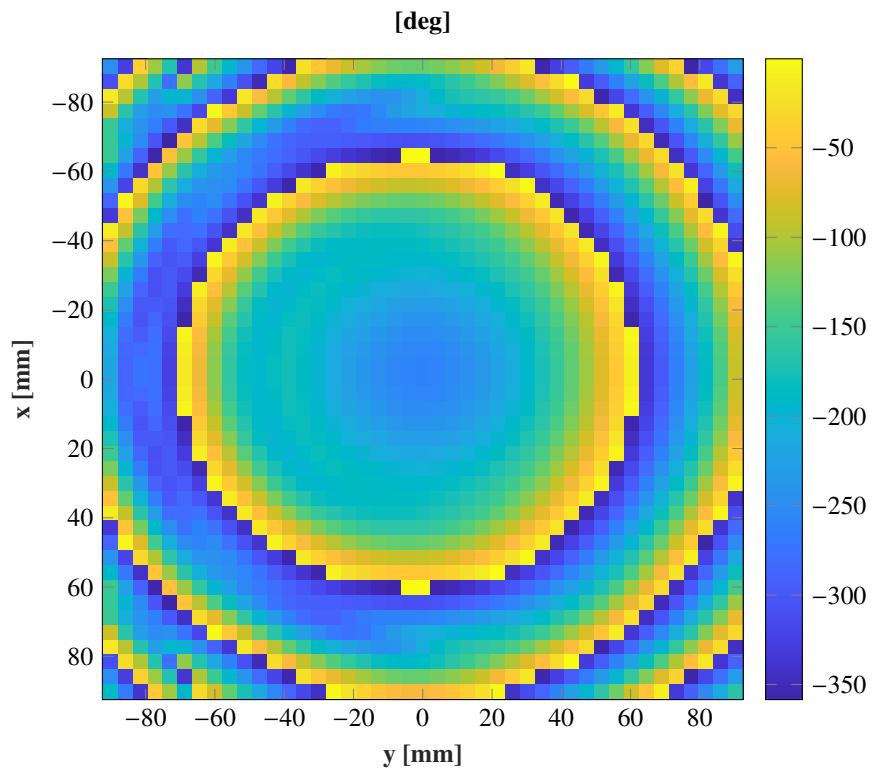

Fig. 8: Optimized phase distribution obtained after the optimization process.

I outlines a comparison of maximum ripples and specification compliance before and after the optimization. Both amplitude and phase show a significant improvement, highlighting the increment from a $25 \%$ to a nearly $90 \%$ of compliance in the amplitude and a ripple reduction of $7 \mathrm{~dB}$. Despite having a markedly better starting point on the phase, the optimized one reduces its ripple to $4.30^{\circ}$ in the whole quiet zone.

Note that the main cuts $(x=0$ and $y=0)$ are of a particular interest since they represent the largest dimension of the quiet zone because of its circular shape, specially the cut $y=0$ that is directly affected by the feed position. After the synthesis this cut has been significantly enlarged with an increase of a $262 \%$ and $303 \%$ in the amplitude and phase quiet zone size, respectively. The cut $x=0$ is also enlarged in a $209 \%$ and $135 \%$ in amplitude and phase respectively. Hence, the current quiet zone has the same size in the main cuts.

Table I: Evaluation of the maximum ripple and the specification compliance in the quiet zone and its main cuts for the starting and optimized point.

\begin{tabular}{cccccc} 
& & \multicolumn{2}{c}{ Starting point } & \multicolumn{2}{c}{ Optimized point } \\
\cline { 3 - 6 } & & $\begin{array}{c}\text { Max. } \\
\text { ripple }\end{array}$ & $\begin{array}{c}\text { Compliance } \\
(\%)\end{array}$ & $\begin{array}{c}\text { Max. } \\
\text { ripple }\end{array}$ & $\begin{array}{c}\text { Compliance } \\
(\%)\end{array}$ \\
\hline \hline Quiet & Ampl. (dB) & -8.01 & 25.25 & -1.33 & 89.42 \\
Zone & Phase $\left(^{\circ}\right)$ & 26.82 & 46.01 & 4.30 & 100 \\
\hline Main & Ampl. (dB) & -8.01 & 38.83 & -1.10 & 83.49 \\
Cuts & Phase $\left(^{\circ}\right)$ & 26.82 & 39.80 & 4.30 & 100 \\
\hline
\end{tabular}

\section{Reflectarray design}

Once the phase distribution on the reflectarray surface has been obtained, the elements of the reflectarray must be designed. In the designing process, the sizes of the printed 


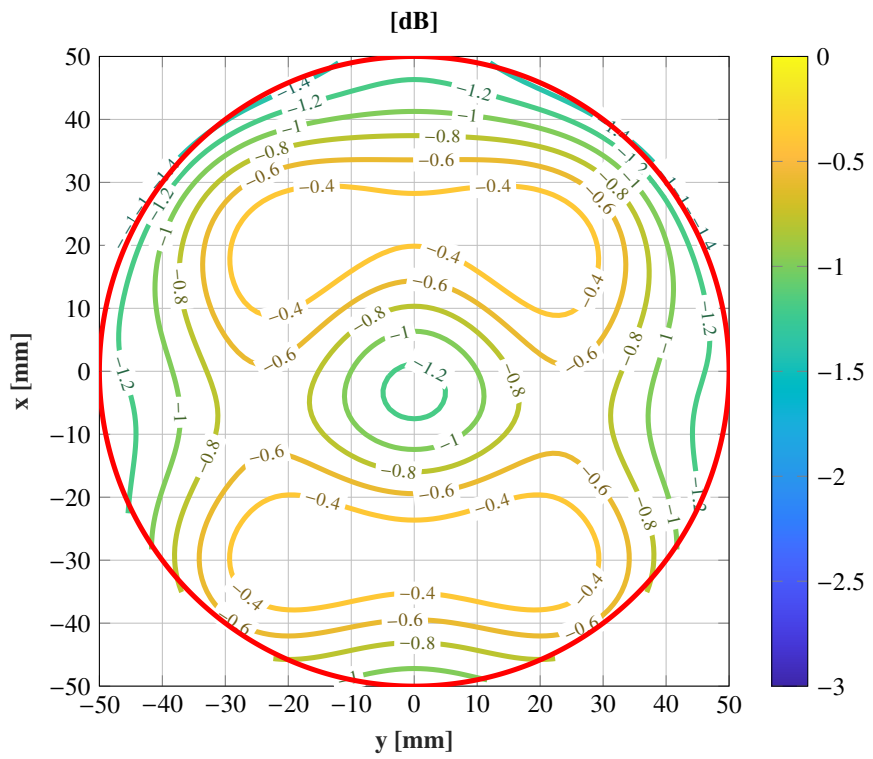

(a)

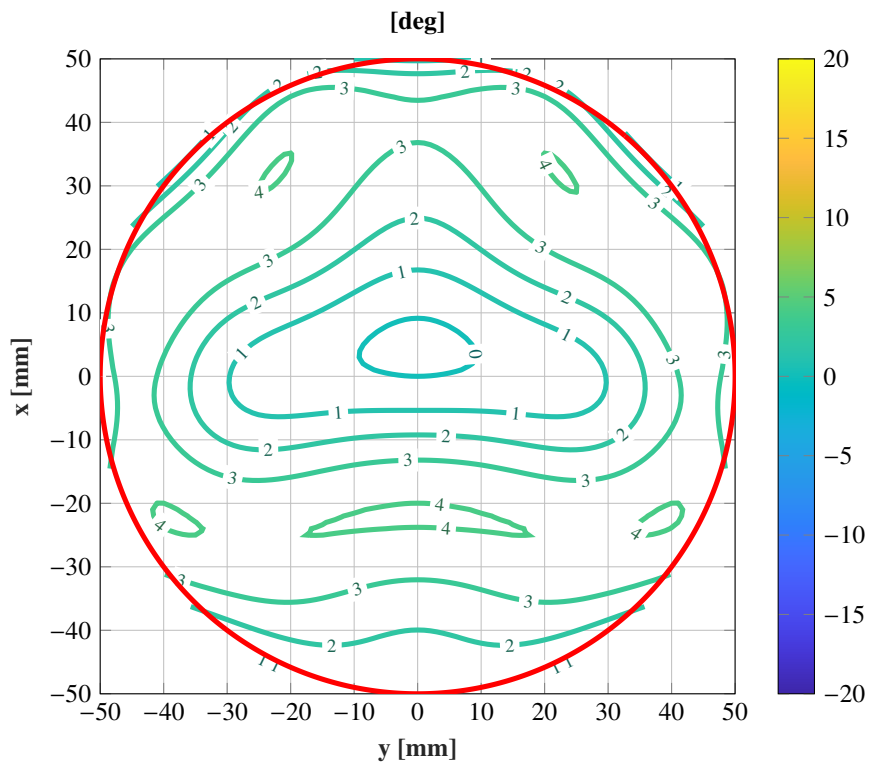

(b)

Fig. 9: Optimized quiet zone. (a) Normalized amplitude (dB) to the maximum. (b) Normalized phase (deg) to the value at the centre of the zone.

dipoles on the surface are adjusted to produce the required phase-shift. In this case, the process is carried out element by element, considering local periodic environment and, the real angle of incidence for each cell. A zero routing [23] is applied to obtain the length $L_{2}$, allowing to match the required phase response of the cell obtained through a home made software based on Method of Moments with local periodicity (LP-MoM) [28] and the synthesized phase in the previous step. The output of the process is the layout of the $44 \times 44$ element reflectarray with a maximum error in the response of $0.1^{\circ}$ for an individual cell.

The near-field radiated by the designed reflectarray has been computed in order to evaluate the performances of the quiet

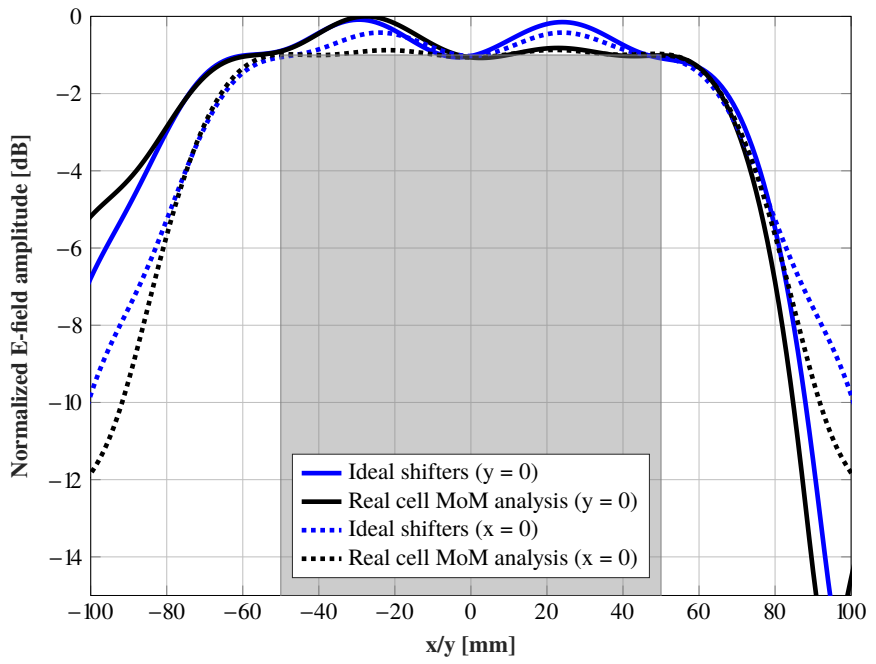

(a)

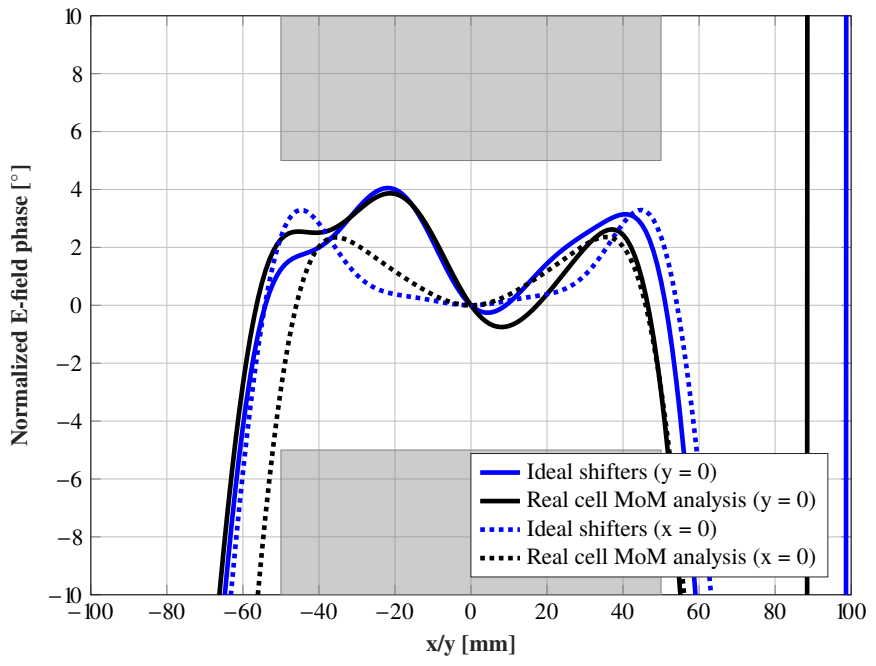

(b)

Fig. 10: Main cuts comparison between the synthesis results and the MoM analysis. (a) Normalized amplitude (dB) to the maximum. (b) Normalized phase (deg) to the value at the centre of the zone.

zone and compare them with the requirements. The resulting quiet zone are shown in Fig. 10, where slight differences can be appreciated, mainly as a consequence of the amplitude losses of the cell that are not considered in the phase-only synthesis, where the reflectarray elements are modelled as ideal phase-shifters. However, MoM results clearly satisfy the specifications within the desired area, showing a suitable quiet zone and validating the reflectarray design.

\section{EXPERIMENTAL VALIDATION}

The squared reflectarray of $44 \times 44$ cells has been manufactured. In order to properly evaluate the quiet zone, the prototype is measured in the planar acquisition range at the University of Oviedo. The reflectarray is placed on an aluminium structure and tilted $\theta_{0}=20^{\circ}$ (see Fig. 11), thus the plane wherein the quiet zone can be found is parallel to the probe aperture (an open-ended Ka-band waveguide). Although 


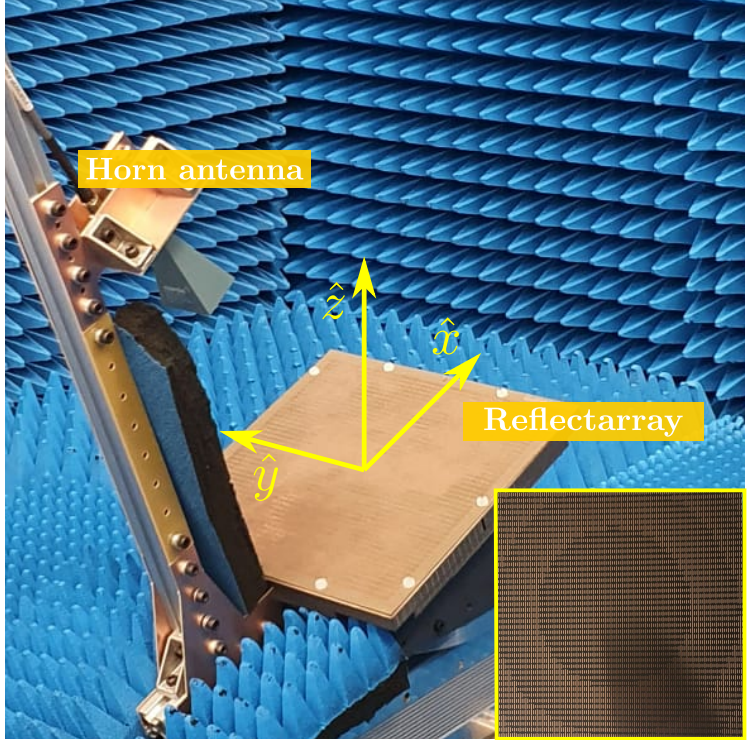

Fig. 11: Set-up at the planar acquisition range and zoom of the reflectarray.

the optimized plane corresponds to $z=500 \mathrm{~mm}$, another three additional planes have been measured at a distance of 550 , 600 and $650 \mathrm{~mm}$. The nearby planes but after the optimized one are also affected though no specifications are imposed on them. Each plane is measured in a regular squared grid of $150 \times 150 \mathrm{~mm}^{2}$ that totally covers the quiet zone. In Fig. 12 the different measured main cuts of the quiet zone in amplitude (a) and phase (b) are shown and the whole area is shown in Fig. 13. All cases show a notably flattened in amplitude and, specially, in phase in most of the desired area as Table II outlines. The table gathers a detailed studio of the maximum peak-to-peak ripple and its percentage of compliance for different specifications levels. The plane at $z=500 \mathrm{~mm}$ has a maximum peak-to-peak ripple, evaluating the whole quiet zone, of $2.07 \mathrm{~dB}$, Fig. 13a, and more than a $93 \%$ is within 1 to $1.5 \mathrm{~dB}$ peak-to-peak ripple. Main cuts show an amplitude taper lower than $1 \mathrm{~dB}$ and a maximum ripple of $1.63 \mathrm{~dB}$ in the worst case $(y=0)$. The maximum phase deviation is $15.33^{\circ}$ and nearly the $90 \%$ of the total area has a peak-to-peak ripple lower than $10^{\circ}$ (see Fig. 13e). The phase along its main cuts is particularly flat with a maximum ripple of $11^{\circ}$. The uniformity of this plane is extended to the measured volume. The plane $z=550 \mathrm{~mm}$ shows a maximum peak-to-peak ripple of $2.15 \mathrm{~dB}$ whilst a $91 \%$ of the area is within a $1.5 \mathrm{~dB}$ peak-to-peak, Fig. 13b. The amplitude taper at its main cuts is lower than $1 \mathrm{~dB}$ and the maximum ripple is $1.75 \mathrm{~dB}$. Although the phase has a singular area that increases the maximum deviation to $19^{\circ}$, the quiet zone phase has a smooth variation, a $93 \%$ has a peak-to-peak ripple lower than $15^{\circ}$ and a $84 \%$ within $10^{\circ}$, Fig. 13f. The amplitude at $z=600 \mathrm{~mm}$ has a similar behaviour to previous planes, having a maximum peak-to-peak ripple of $2.43 \mathrm{~dB}$, noticing that practically the $90 \%$ of the total quiet zone is within a 1.5 $\mathrm{dB}$ ripple, Fig. 13c. The amplitude taper in both main cuts is lower than $1 \mathrm{~dB}$ and its maximum ripple is $1.69 \mathrm{~dB}$. The

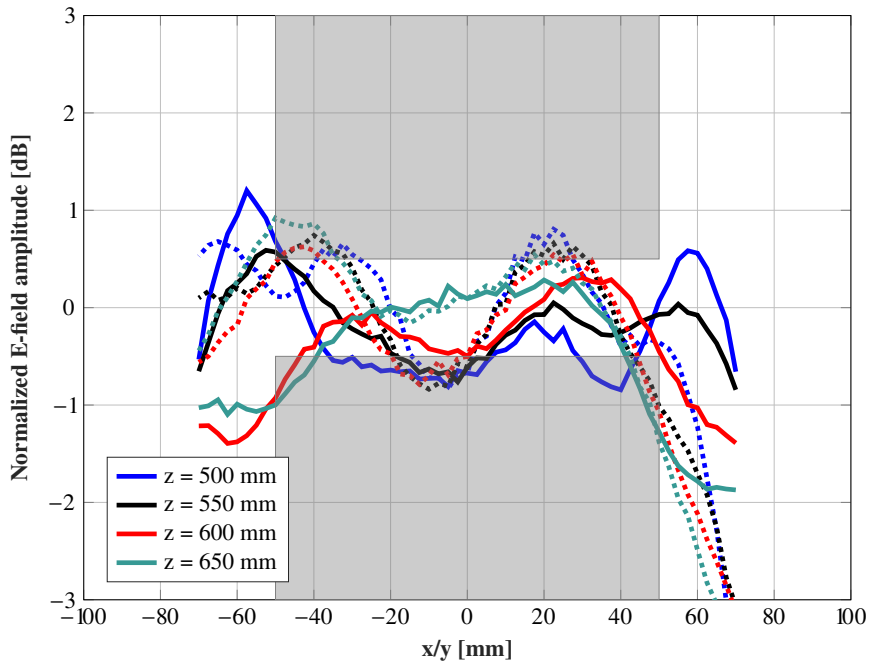

(a)

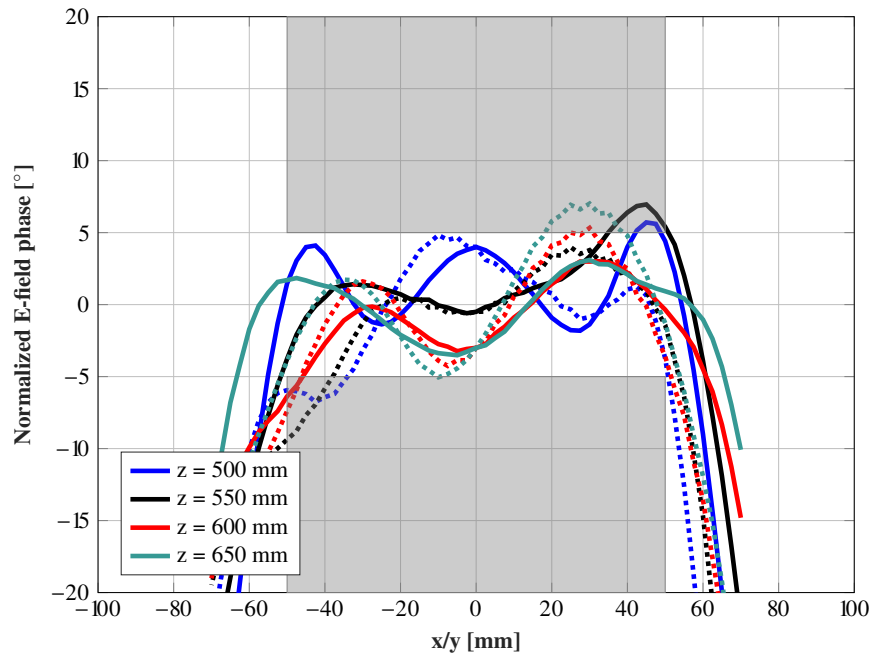

(b)

Fig. 12: Main cuts of the measurements at $28 \mathrm{GHz}$ (a) Normalized amplitude $(\mathrm{dB})$ to the mean value of the quiet zone. (b) Normalized phase (deg) to the value mean value of the zone (solid line) cut $y=0$ (dotted line) cut $x=0$.

phase at this plane is markedly flat, the maximum deviation is close to the $15^{\circ}$ of the plane $z=500 \mathrm{~mm}$, and a maximum ripple of $10^{\circ}$ in more than the $88 \%$ of the area, Fig. 13g. The plane $z=650 \mathrm{~mm}$ slightly increases the peak-to-peak ripple to $3 \mathrm{~dB}$, and, a $83 \%$ of the quiet zone is within a 1.5 ripple. However, only the upper edge of the quiet zone is affected by this increase (see Fig. 13d). The central area remains with a very smooth variation close to $0.8 \mathrm{~dB}$ ripple. The amplitude taper in both main cuts is close to $1 \mathrm{~dB}$, though the ripple is higher than other planes. The uniformity of the phase is undoubtedly smooth, nearly the $90 \%$ is under a $10^{\circ}$ peak-topeak ripple and the whole area within $15^{\circ}$, Fig. $13 \mathrm{~h}$.

\section{CONCLUSIONS}

A reflectarray antenna made of cells based on three parallel dipoles is proposed to be used as a probe in a compact and portable CATR for $5 \mathrm{G}$ new radio devices measurements. 
Table II: Evaluation of the maximum ripple and the specification compliance in the measured quiet zone and its main cuts.

\begin{tabular}{|c|c|c|c|c|c|c|c|c|c|}
\hline & \multirow[b]{2}{*}{ Plane } & \multicolumn{4}{|c|}{ Amplitude } & \multicolumn{4}{|c|}{ Phase } \\
\hline & & $\begin{array}{l}\text { Max. peak-to-peak } \\
(\mathrm{dB})\end{array}$ & $\begin{array}{c}1 \mathrm{~dB} \\
\text { Compliance } \\
(\%)\end{array}$ & $\begin{array}{c}1.2 \mathrm{~dB} \\
\text { Compliance } \\
(\%)\end{array}$ & $\begin{array}{c}1.5 \mathrm{~dB} \\
\text { Compliance } \\
(\%)\end{array}$ & $\begin{array}{l}\text { Max. deviation } \\
\left({ }^{\circ}\right)\end{array}$ & $\begin{array}{c}10^{\circ} \\
\text { Compliance } \\
(\%)\end{array}$ & $\begin{array}{c}15^{\circ} \\
\text { Compliance } \\
(\%)\end{array}$ & $\begin{array}{c}20^{\circ} \\
\text { Compliance } \\
(\%)\end{array}$ \\
\hline \multirow{4}{*}{$\begin{array}{l}\text { Quiet } \\
\text { zone }\end{array}$} & $500 \mathrm{~mm}$ & -2.07 & 73.11 & 83.09 & 93.22 & 15.33 & 88.88 & 95.35 & 100 \\
\hline & $550 \mathrm{~mm}$ & -2.15 & 71.05 & 81.34 & 91.16 & 19.93 & 84.23 & 93.67 & 97.18 \\
\hline & $600 \mathrm{~mm}$ & -2.43 & 73.35 & 82.63 & 89.64 & 15.91 & 88.04 & 97.79 & 100 \\
\hline & $650 \mathrm{~mm}$ & -3.02 & 63.44 & 71.28 & 83.01 & 13.19 & 88.49 & 100 & 100 \\
\hline \multirow{4}{*}{$\begin{array}{l}\text { Cut } \\
\mathrm{y}=0\end{array}$} & $500 \mathrm{~mm}$ & -1.63 & 63.41 & 75.06 & 92.68 & 11.58 & 82.90 & 100 & 100 \\
\hline & $550 \mathrm{~mm}$ & -1.75 & 43.90 & 63.41 & 85.36 & 13.36 & 82.92 & 97.56 & 100 \\
\hline & $600 \mathrm{~mm}$ & -1.69 & 58.53 & 78.04 & 92.68 & 12.70 & 90.24 & 100 & 100 \\
\hline & $650 \mathrm{~mm}$ & -2.30 & 70.73 & 78.04 & 78.05 & 12.05 & 73.17 & 100 & 100 \\
\hline
\end{tabular}

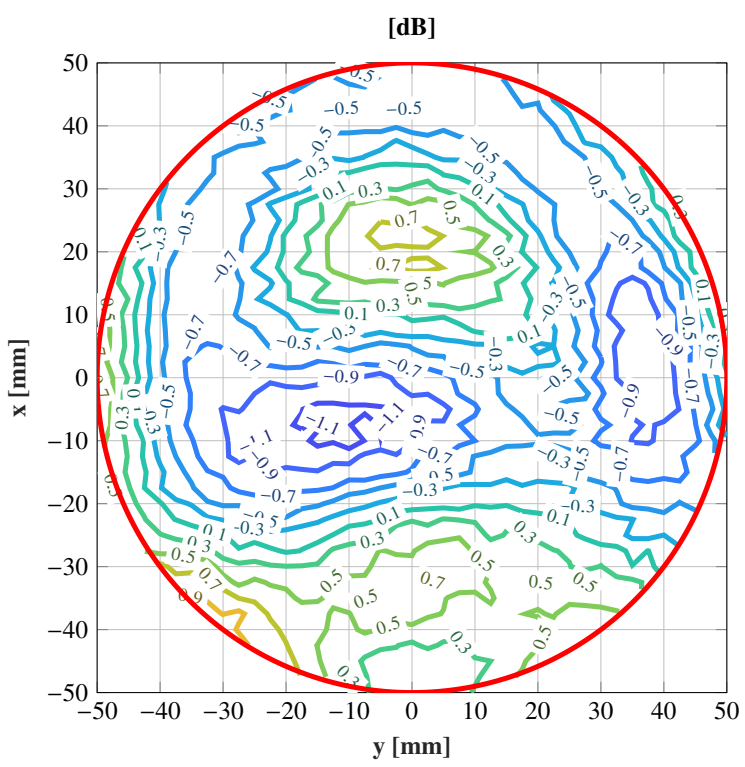

(a)

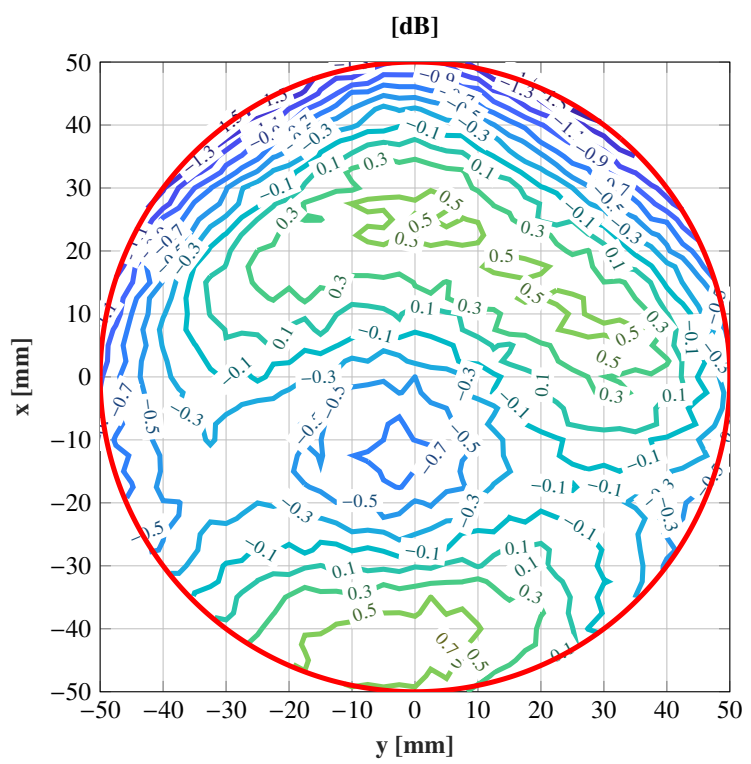

(c)
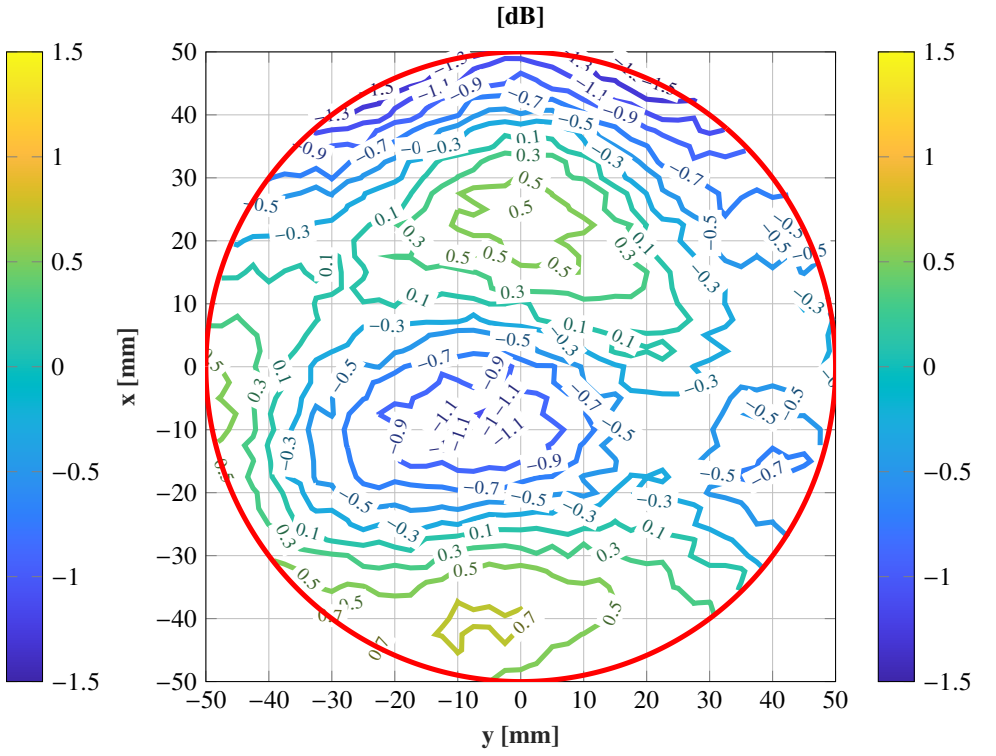

(b)

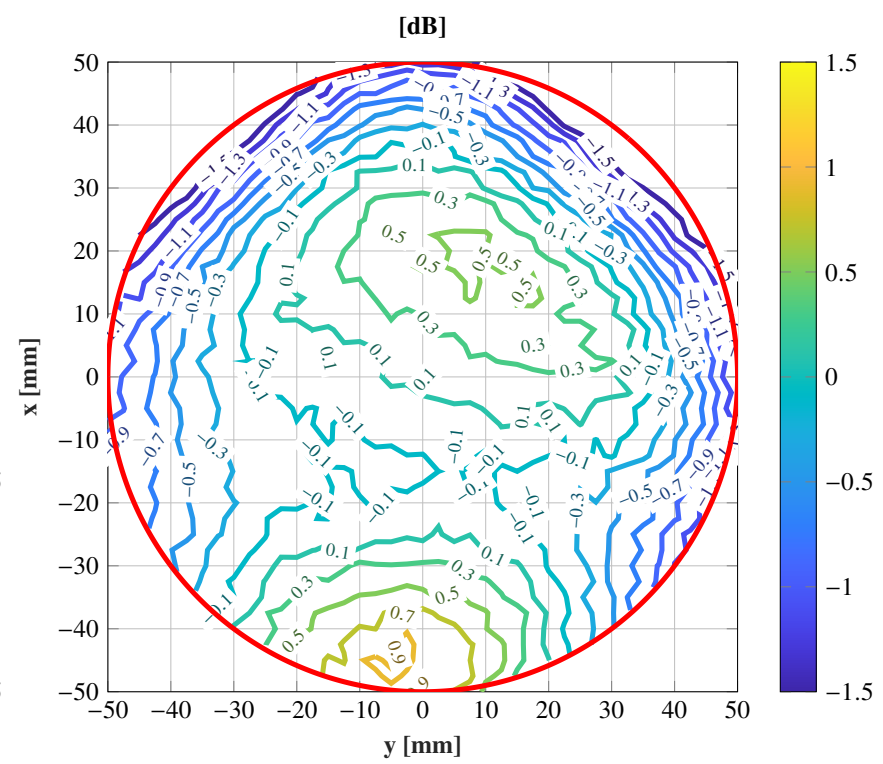

(d) 


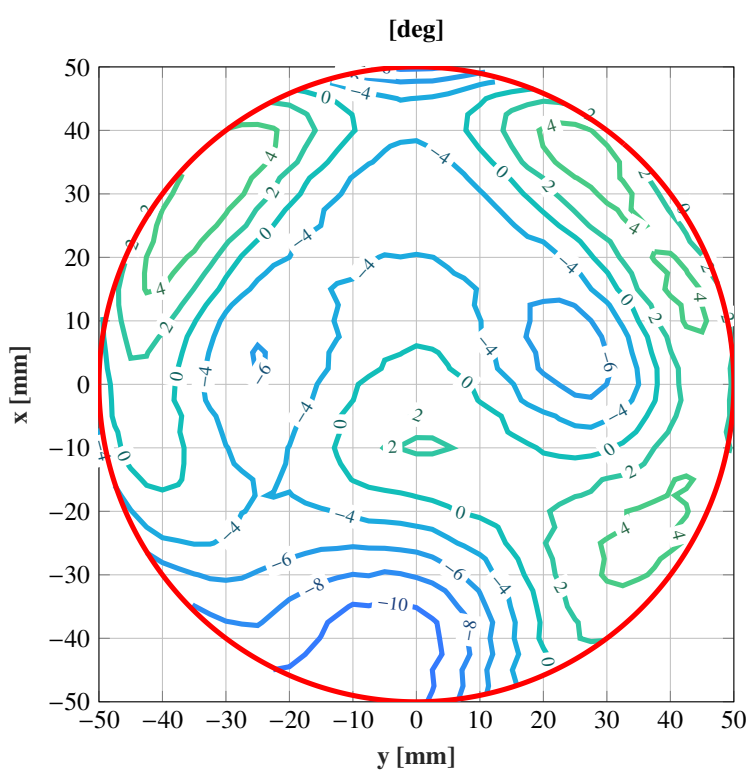

(e)

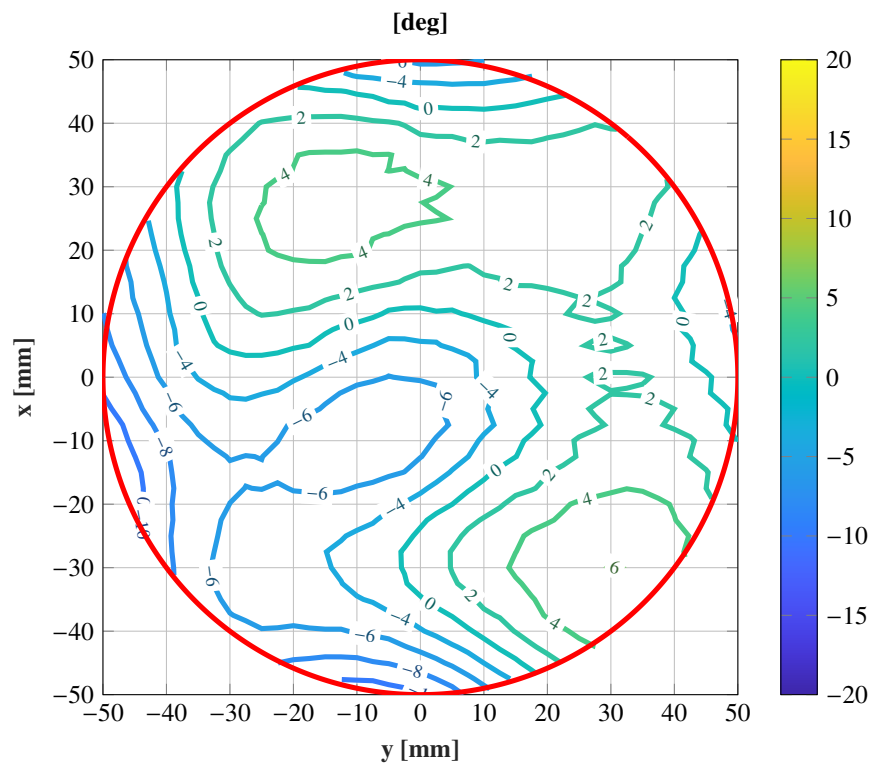

(g)

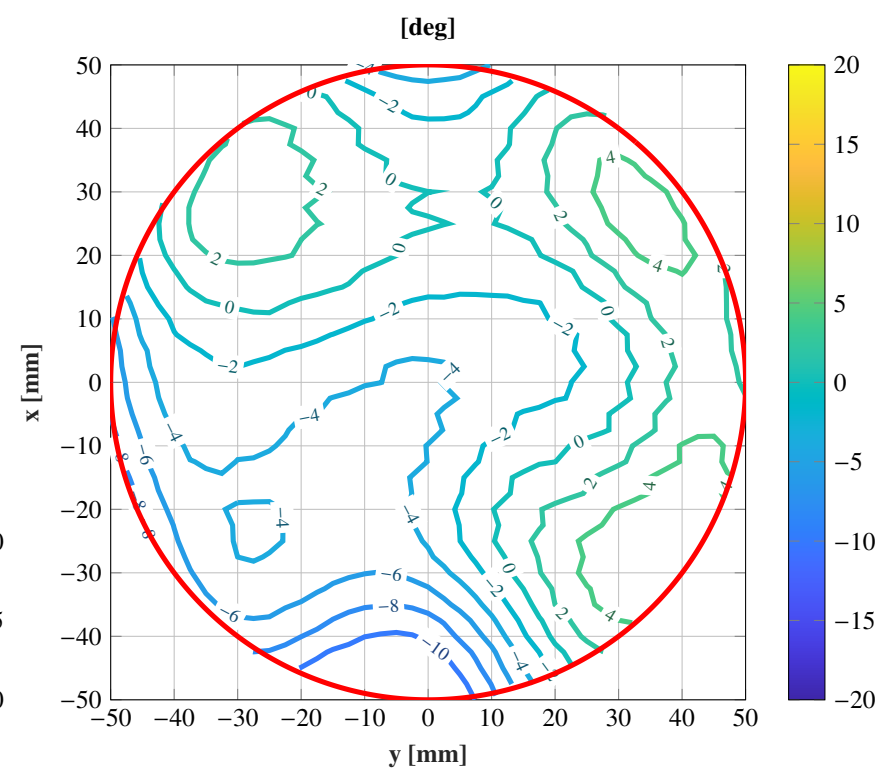

(f)

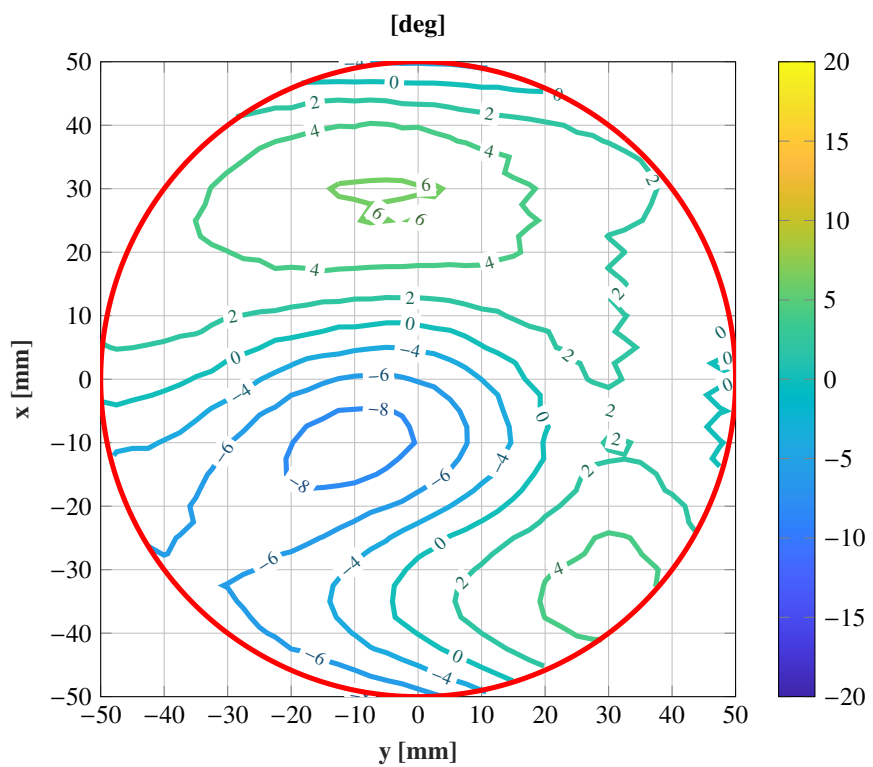

(h)

Fig. 13: Quiet zone measurements of the planes $z=500, z=550, z=600$ and $z=650 \mathrm{~mm}$ at $28 \mathrm{GHz}$. (a) Normalized amplitude (dB) at $z=500 \mathrm{~mm}(\mathrm{~b})$ Normalized amplitude (dB) at $z=550 \mathrm{~mm}$ (c) Normalized amplitude (dB) at $z=600$ $\mathrm{mm}$ (d) Normalized amplitude (dB) at $z=650 \mathrm{~mm}$ (e) Normalized phase (deg) at $z=500 \mathrm{~mm}$ (f) Normalized phase (deg) at $z=550 \mathrm{~mm}(\mathrm{~g})$ Normalized phase (deg) at $z=600 \mathrm{~mm}(\mathrm{~h})$ Normalized phase (deg) at $z=650 \mathrm{~mm}$. Both amplitude and phase are normalized to the mean value of each quiet zone.

Particularly, a reflectarray comprised by $44 \times 44$ elements with an equivalent aperture of $177.36 \times 188.76 \mathrm{~mm}^{2}$. This antenna should generate a quiet zone height of $100 \mathrm{~mm}\left(56 \%\right.$ of $\left.D_{x}\right)$, a width of $100 \mathrm{~mm}\left(53 \%\right.$ of $D_{y}$ ) and a depth of $150 \mathrm{~mm}$ starting at a distance of $500 \mathrm{~mm}(46.6 \lambda)$ from the center of the antenna. Considering that the far-field starts at $12.54 \mathrm{~m}(1170.40 \lambda)$, a quite compact structure is achieved at a working frequency of $28 \mathrm{GHz}$. A synthesis technique for the near-field radiated by a reflectarray using amplitude and phase constraints is presented for the first time. In this case, the generalized Intersection Approach is employed to synthesize both amplitude and phase of the near-field. In order to avoid the use of a backward propagating model, the LMA is integrated inside the algorithm working as its backward propagation. The synthesis results have been obtained optimizing only the phase of the reflection coefficients of the reflectarray elements. The provided solution nearly reaches the $1 \mathrm{~dB}$ and $10^{\circ}$ ripple specification in the desired circular area of $100 \mathrm{~mm}$ of diameter. Additionally, 
the phase distribution along the reflectarray surface is smooth enough to perform a design. Particularly, a reflectarray cell based on three parallel dipoles has been chosen, allowing to cover more than $360^{\circ}$ of the required phase-shift with a low level of losses. The design is analysed using a MoM software and a high agreement with synthesis results is obtained. Thus, it is manufactured and this prototype is measured in a planar range facility in order to evaluate the generated quiet zone. Four different planes have been measured, starting from the optimized plane to a total distance of $150 \mathrm{~mm}$. Measurements show a high percentage of the quiet zone satisfying the $1 \mathrm{~dB}$ ripple in amplitude and the $10^{\circ}$ in phase. A nearly $90 \%$ of the measured planes is within 1 to $1.5 \mathrm{~dB}$ peak-to-peak ripple and a taper lower than $1 \mathrm{~dB}$, evaluating the main cuts of the quiet zone. The phase is significantly constant along the measured volume and almost a $90 \%$ is within $10^{\circ}$ and the $100 \%$ within $15^{\circ}$. Hence, the prototype practically satisfies the theoretical quiet zone specifications in a quiet zone of size $100 \times 100 \times 150$ $\mathrm{mm}$. In the light of these results, reflectarray antennas have demonstrated to be a potential candidate in the generation of quiet zones at high frequencies, taking the advantage of improving the quiet zone performances using the near-field technique presented in this work.

\section{REFERENCES}

[1] C. A. Balanis, "Antenna theory: analysis and design," in Hoboken., NJ, USA: John Wiley \& Sons, 2005.

[2] A. D. Olver, "Compact antenna test ranges," in Proc. 7th Int. Conf. Antennas and Propagation (ICAP), York, UK, 1991, pp.99-108.

[3] C. Mao, M. Khalily, P. Xiao, T. W. Brown, and S. Gao, "Planar submillimeter-wave array antenna with enhanced gain and reduced sidelobes for 5G broadcast applications," in IEEE Trans. Antennas Propag., vol. 67, no. 1, pp. 160-168, Jan., 2019.

[4] A. F. Vaquero, M. Arrebola, M. R. Pino, "Spatially-fed arrays for nearfield multiple-spot coverage," in International Conf. on Electro. in Advanced App. (ICEAA), Granada, Spain, Sept., 2019.

[5] N. Nachabe, C. Luxey, D. Titz, J. R. Costa, S. A. Matos, F. Gianesello, and C. A. Fernandes "Low-cost $60 \mathrm{GHz} 3 \mathrm{D}$ printed lens fed by a planar source with WR15 transition integrated on FR4 PCB," in Proc. IEEE International Symposium on Antennas and Propagation \& USNC/URSI National Radio Science Meeting (APS), San Diego, CA, USA, Jul., 2017.

[6] C. G. Salzburg, T. Vaupel, T. Bertuch, M. Wilhelm, T. Wichmann, and S. T. Alfageme, "Feasibility of an automotive radar antenna at $77 \mathrm{GHz}$ on LTCC substrate," IET Radar, Sonar \& Navigation, vol. 12, no. 10, pp. 1172-1178, Sep., 2018.

[7] S. B. Yeap, X. Qing, and Z. N. Chen, "77-GHz dual-layer transmit-array for automotive radar applications," IEEE Trans. Antennas Propag., vol. 63, no. 6, pp. 2833-2837, Apr., 2015.

[8] P. Naseri, S. A. Matos, J. R. Costa, C. A. Fernandes, and N. J. G. Fonseca, "Dual-band dual-linear-to-circular polarization converter in transmission mode application to $\mathrm{K} / \mathrm{Ka}$-band satellite communications," in IEEE Trans. Antennas Propag., vol. 66, no. 12, pp. 7128-7137, Dec., 2018.

[9] D. Martinez-De-Rioja, E. Martinez-De-Rioja, J. A. Encinar, R. Florencio, G. Toso, "Reflectarray to generate four adjacent beams per feed for multispot satellite antennas," in IEEE Trans. Antennas Propag., vol. 67, no. 2, pp. 1265-1269, Feb., 2019.

[10] A.D. Olver, and A.A. Saleeb, "Lens-type compact antenna test range," Electron. Lett., vol. 15, no. 14, pp. 409-1979, 1979.

[11] T. Hirvonen, J. Tuovinen, and A. Räisänen, "Lens-type compact antenna test range at mm-waves," in Proc. 21st Eur. Microwave Conf., Stuttgart, Germany, pp. 1079-1083, Sept., 1991.

[12] J. Tuovinen, A. Vasara and A. Räisänen, "A new type of compact antenna test range," in Proc. 22nd Eur. Microwave Conf., Helsinki, Finland, pp. 503-508, Sept., 1992.

[13] T. Sehm, J. Ala-Laurinaho, T. Hirvonen and A. V. Räisänen, "Antenna measurements using a hologram CATR," Electron. Lett., vol. 35, no. 10, pp. 757-758, May., 2005.
[14] J. Ala-Laurinaho, T. Hirvonen, P. Piironen, A. Lehto, J. Tuovinen, A. V. Räisänen, and U. Frisk, "Measurement of the Odin telescope at $119 \mathrm{GHz}$ with a hologram-type CATR," IEEE Trans. Antennas Propag., vol. 49, no. 9, pp. 1264-1270, Sept., 2001.

[15] D. R. Prado, M. Arrebola, M. R. Pino, R. Florencio, R. R. Boix, J. A. Encinar, and F. Las-Heras, "Support vector regression to accelerate design and crosspolar optimization of shaped-beam reflectarray antennas for space applications," IEEE Trans. Antennas Propag., vol. 67, no. 3, pp. 1659-1668, Mar., 2019.

[16] D. R. Prado , M. Arrebola, M. R. Pino, "Reflectarray pattern optimization for advanced wireless communications", in Array pattern optimization, E. Aksoy, Ed. London: IntechOpen, 2019, ch. X, pp. 121., DOI: 10.5772/intechopen.88909. ISBN 978-1-83880-108-3.

[17] A. F. Vaquero, D. R. Prado, M. Arrebola, and M. R. Pino, "Reflectarray antennas for 5-G indoor coverage," in Proc. 13rd Eur. Conf. Antennas Propag. (EuCAP), Krakow, Poland, Mar., 2019.

[18] D. R. Prado, A. F. Vaquero, M. Arrebola, M. R. Pino, and F. Las-Heras, "General near field synthesis of reflectarray antennas for their use as probes in CATR," Prog. Electromagn. Res., vol. 160, pp. 9-17, Oct., 2017.

[19] D. R. Prado, M. Arrebola, M. R. Pino, R. Florencio, R. R. Boix, J. A. Encinar, and F. Las-Heras, "Efficient crosspolar optimization of shaped-beam dual-polarized reflectarrays using full-wave analysis for the antenna element characterization," IEEE Trans. Antennas Propag., vol. 65, no. 2, pp. 623-635, Dec., 2019.

[20] D. R. Prado, J. A. López Fernández, M. Arrebola, M. R. Pino, and G. Goussetis, "General Framework for the Efficient Optimization of Reflectarray Antennas for Contoured Beam Space Applications," IEEE Access, pp. 72295-72310, Nov., 2018.

[21] A. F. Vaquero, D. R. Prado, M. Arrebola, M. R. Pino, and F. Las-Heras, "Near field synthesis of reflectarrays using Intersection Approach," in Proc. 11st Eur. Conf. Antennas Propag. (EuCAP), Paris, France, Mar. 19-24, 2017.

[22] R. Florencio, J. A. Encinar, R. R. Boix, and G. Perez-Palomino, "Dualpolarization reflectarray made of cells with orthogonal sets of parallel dipoles for bandwidth and cross-polarisation improvement," in IET Microw. Ant. \& Propag., vol. 8, no. 15, pp. 1389-1397, Dic. 2014.

[23] J. Huang, and J. A. Encinar, "Reflectarray antennas," in Hoboken., NJ, USA: John Wiley \& Sons, 2008.

[24] D. R. Prado, M. Arrebola, M. R. Pino, and F. Las-Heras, "Evaluation of the quiet zone generated by a reflectarray," in International Conf. on Electro. in Advanced App. (ICEAA), Cape Town, South Africa, Sept., 2012.

[25] O. M. Bucci, G. D’Elia, G. Mazzarella, and G. Panariello, "Antenna pattern synthesis: A new general approach," Proc. IEEE, vol. 82, no. 3, pp. 358-371, Mar., 1994.

[26] D. R. Prado, A. F. Vaquero, M. Arrebola, M. R. Pino, and F. Las-Heras, "Acceleration of gradient-based algorithms for array synthesis with farfield or near-field constraints," IEEE Trans. Antennas Propag., vol. 66, no. 10 , pp. 5239-5248, Oct., 2018.

[27] D. R. Prado, J. López-Fernández, M. Arrebola, M. R. Pino, and G. Goussetis, "Comparison of different approaches in reflectarrays synthesis based on Intersection Approach," in International Conf. on Electro. in Advanced App. (ICEAA), Granada, Spain, 9-13 Sept., 2019.

[28] R. Florencio, R. R. Boix, E. Carrasco, J. A. Encinar, and V. Losada, "Efficient numerical tool for the analysis and design of reflectarrays based on cells with three parallel dipoles," in Microw. Opt. Technol. Lett., vol. 55, no. 6, pp. 1212-1216, Jun. 2013. 


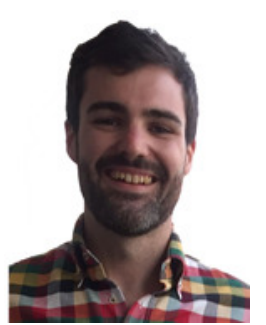

Álvaro F. Vaquero was born in Salinas, Spain, in 1990. He received the B.Sc. and M.Sc. degrees in telecommunications engineering from the University of Oviedo, Gijón, Spain, in 2015 and 2107, respectively, where he is currently pursuing the Ph.D. degree.

Since 2016, he has been a Research Assistant with the Signal Theory and Communications Area, University of Oviedo. In 2017, he was with the Instituto de Telecomunicações, Lisbon, Portugal, where he was involved in the design of broadband planar lenses for skin cancer imaging. His current research interests include the development of efficient techniques for the analysis and synthesis of reflectarrays and tansmitarrays antennas and the design of 3-D printed dielectric lenses for near-field applications.

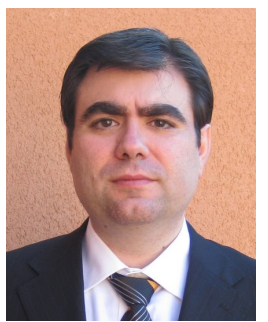

Manuel Arrebola (S'99-M'02-SM'17)was born in Lucena (Córdoba), Spain. He received the M.Sc. degree in telecommunication engineering from the University of Málaga, Málaga, Spain, in 2002, and the Ph.D. degree from the Technical University of Madrid (UPM), Madrid, Spain, in 2008.

From 2003 to 2007, he was a Research Assistant with the Electromagnetism and Circuit Theory Department, UPM. In 2005, he was a Visiting Scholar with the Microwave Techniques Department, Universität Ulm, Ulm, Germany. In 2007, he joined the Electrical Engineering Department, University of Oviedo, Gijón, Spain, where he is currently an Associate Professor. In 2009, he enjoyed a two-month stay at European Space Research and Technology Centre, European Space Agency, Noordwijk, The Netherlands. In 2018, he was a Visiting Professor with the Edward S. Rogers Sr. Department of Electrical and Computer Engineering, University of Toronto, Toronto, ON, Canada. In 2019, he was a Visiting Professor with the Institute of Sensors, Signals and Systems, HeriotWatt University, Edinburgh, UK. His current research interests include the development of efficient analysis, design, and optimization techniques of reflectarray and transtmitarray antennas both in near and far fields.

Dr. Arrebola was a co-recipient of the 2007 S. A. Schelkunoff Transactions Prize Paper Award by the IEEE Antennas and Propagation Society.

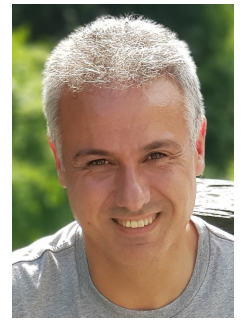

Marcos R. Pino was born in Vigo, Spain, in 1972. He received the M.Sc. and Ph.D. degrees in telecommunication engineering from the University of Vigo, Vigo, in 1997 and 2000, respectively. In 1998, he was a Visiting Scholar with the ElectroScience Laboratory, The Ohio State University, Columbus, OH, USA. From 2000 to 2001, he was Assistant Professor with the University of Vigo. Since 2001, he has been with the Electrical Engineering Department, University of Oviedo at Gijón, Gijón, Spain, where he is currently an Associate Professor, teaching courses on communication systems and antenna design. From 2017 to 2019, he has spent several months as Visiting Fellow with the Department of Information Engineering, University of Pisa, Italy. His current research interests include antenna design, measurement techniques, and efficient computational techniques applied to EM problems, such as evaluation of radar cross section or scattering from rough surfaces.

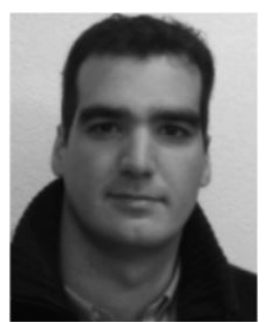

Rafael Florencio received the Licenciado degree in physics from the University of Seville, Seville, Spain, in 2008, and the master's degree in communications technology and systems and the Ph.D. degree in telecommunications engineering from the Universidad Politécnica de Madrid, Madrid, Spain, in 2010 and 2016, respectively. His current research interests include the efficient design of dual-polarization, dual-frequency reflectarray antennas for satellite applications.

Dr. Florencio was a recipient of the Scholarship financed by the "Junta de Andalucía" to carry out his Ph.D. dissertation.

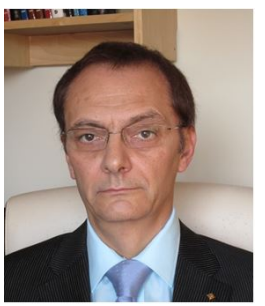

José A. Encinar (Fellow, IEEE) was born in Madrid, Spain. He received the Electrical Engineer and Ph.D. degrees, both from Universidad Politécnica de Madrid (UPM), in 1979 and 1985, respectively.

Since January 1980, he has been with the Applied Electromagnetics Group at UPM, as a Teaching and Research Assistant from 1980 to 1982, as an Assistant Professor from 1983 to 1986, and as Associate Professor from 1986 to 1991. From February to October of 1987, he was a Postdoctoral Fellow of the NATO Science Program with the Polytechnic University, Brooklyn, NY, USA. Since 1991 he is a Professor of the Electromagnetism and Circuit Theory Group, currently in the Department of Signals, Systems and Radio Communications at UPM. He was a Visiting Professor with the Laboratory of Electromagnetics and Acoustics at Ecole Polytechnique Fédérale de Lausanne (EPFL), Switzerland, in 1996, and with the Institute of Electronics, Communication and Information Technology (ECIT), Queen's University Belfast, U.K., in 2006 and 2011. His research interests include numerical techniques for the analysis of multi-layer periodic structures, design of frequency selective surfaces, printed arrays and reflectarrays.

Prof. Encinar has co-authored more than 200 journal and conference papers, one book and several book chapters. He is holder of five patents on array and reflectarray antennas. He was a co-recipient of the $2005 \mathrm{H}$. A. Wheeler Applications Prize Paper Award and the 2007 S. A. Schelkunoff Transactions Prize Paper Award, given by IEEE Antennas and Propagation Society. He has been a member of the Technical Programme Comity of several International Conferences (European Conference on Antennas and Propagation, ESA Antenna Workshops, Loughborough Antennas \& Propagation Conference). 